\title{
Homogeneous Perturbed Classical States and Equilibrium Conditions
}

\author{
B. Demoen and A. Verbeure \\ Instituut voor Theoretische Fysica, Universiteit Leuven, B-3030 Leuven, Belgium
}

\begin{abstract}
For infinite classical systems a class of spatially homogeneous perturbed states is defined, and used to give a direct proof of the fact that global thermodynamical stability implies the (classical) KMS-condition.
\end{abstract}

\section{§1. Introduction}

The classical KMS-condition or the equilibrium condition on the state of a classical finite system is known for a long time (see e.g. [1]). It is however only recently $[2,3]$ that it has been studied as equilibrium condition for an infinite system. This equation for an equilibrium state should be supported by its connection to properties of stability. It has already been proved [4] that dynamical stability, supplemented with cluster properties leads to KMS-states, exactly as in the quantum mechanical case [5]. It is known that the variational principle for the free energy, yields the KMS-property.

This property can be seen to hold true, via an indirect proof, through D.L.R. equations, Kirkwood-Salzburg equations under some conditions $[2,3,5,6,7]$. In this paper we close the circle by giving a direct proof which gives itself an insight in the physical mechanisms which are involved, in particular we present the variational principle as a notion of what could be called thermodynamical stability.

We formulate the variational principle for infinite classical systems under some conditions which are general enough to include the results of [8] and [9]. The main point is now that we give explicitly a class of states of the system which are spatially homogeneous, slightly perturbed from the equilibrium states in a dissipative way. The physical idea behind this dissipative perturbations, in contradistinction with automorphic perturbations, (see the notion of dynamical stability in $[4,5]$ ) is that the system can be looked upon as a subsystem in interaction with a heat bath. As such the variational principal becomes then a notion of thermodynamical stability or "open system stability", exactly as in the quantum mechanical case $[10,11]$. We do not give the perturbations in terms of a semigroup of positive, unity perserving maps of the algebra into itself, as in the quantum case, but we perturb the states. 


\section{§2. Homogeneous Perturbed States}

First we introduce the algebra of observables and a set of states associated with a family of density distributions as in [12].

The algebra, a suitable class of functions on the phase space, is not at all unique. We choose the algebra of [13] which is generated by the exponential of a class of testfunctions.

The use of this algebra was essential in [3]. We denote by $\Lambda$ always a bounded open set of $\mathbb{R}^{v}, v \in \mathbb{N}$. Let $\mathscr{K}$ be the set of infinite countable subsets $x$ of $\mathbb{R}^{v} \times \mathbb{R}^{v}$ such that $x \cap \Lambda \times \mathbb{R}^{v}$ contains only a finite number of elements. $\mathscr{K}$ is called the set of configurations. Let $\mathcal{O}$ be the set of real $C^{\infty}$-functions of compact support in $\mathbb{R}^{v} \times \mathbb{R}^{v}$. For each element $f \in \mathcal{O}$ define the function $S f$ from $\mathscr{K}$ to $\mathbb{R}$ by: for $x=\left(x_{i}\right)_{i=1}^{\infty}=\left(q_{i}, p_{i}\right)_{i=1}^{\infty} \in \mathscr{K}$,

$$
(S f)(x)=\sum_{i} f\left(x_{i}\right)
$$

We denote by $W(f)$ the bounded function on $\mathscr{K}$ defined by

$$
W(f)=\exp i S f .
$$

Finally the algebra of observables $\mathscr{K}$ is the abelian algebra generated by the set $\{W(f) \mid f \in \mathcal{O}\}$.

Now we consider a subset of states $\mathscr{F}$. For every $\Lambda \subset \mathbb{R}^{v}$ and $n \in \mathbb{N}$, let $\mu_{\Lambda}^{n}$ be a positive measure on $\left(\Lambda \times \mathbb{R}^{v}\right)^{n}$, symmetric in its arguments; $\left(\mu_{\Lambda}^{n}\right)_{n=0}^{\infty}$ is a family of density distributions, if the following conditions are satisfied:

a) normalization:

$$
\sum_{n=0}^{\infty} \mu_{\Lambda}^{n}\left(\left(\Lambda \times \mathbb{R}^{v}\right)^{n}\right)=1
$$

b) compatibility:

$$
\text { if } f \in \mathcal{O}
$$

with support in $\Lambda \times \mathbb{R}^{v}$ then for all $\Lambda^{\prime} \supset \Lambda$

$$
\sum_{n=0}^{\infty} \int d \mu_{\Lambda^{\prime}}^{n}\left(x_{1}, \ldots, x_{n}\right) e^{i \sum_{k=1}^{n} f\left(x_{k}\right)}=\sum_{n=0}^{\infty} \int d \mu_{\Lambda}^{n}\left(x_{1}, \ldots, x_{n}\right) e^{i \sum_{k=1}^{n} f\left(x_{k}\right)}
$$

Given any family $\left(\mu_{A}^{n}\right)$, a state $\omega$ on $\mathscr{A}$ is determined as follows: for each $f \in \mathcal{O}$ with support in $\Lambda \times \mathbb{R}^{v}$

$$
\omega(W(f))=\sum_{n \geqq 0} \int d \mu_{\Lambda}^{n}\left(x_{1}, \ldots, x_{n}\right) \exp i \sum_{k=1}^{n} f\left(x_{k}\right) .
$$

The set of states obtained in this way is denoted by $\mathscr{F}$. A subset $\tilde{\mathscr{F}}$ of $\mathscr{F}$ is the set of states satisfying moreover the following conditions:

(i) The measures $\mu_{\Lambda}^{n}$ are absolutely continuous with respect to the Lebesgue measure, with differentiable weight functions $\sigma_{\Lambda}^{n}$ i.e.

$$
d \mu_{\Lambda}^{n}(X)=\frac{1}{n !} \sigma_{\Lambda}^{n}(X) d X
$$


where $X=\left(x_{1}, \ldots, x_{n}\right), d X=d x_{1} \ldots d x_{n}$.

(ii) The states are translation invariant i.e. for all $a \in \mathbb{R}^{v}$

$$
\sigma_{\Lambda}^{n}(X)=\sigma_{\Lambda+a}^{n}(X+a)
$$

where $X+a$ is a shorthand notation for $\left(q_{1}+a, p_{1}, \ldots, q_{n}+a, p_{n}\right)$.

(iii) For $f=\left(f^{1}, f^{2}, \ldots, f^{k}\right)$ where the $f^{n}$ are real bounded differentiable functions on $\left(\mathbb{R}^{2 v}\right)^{n}$ with $q$-support in $\Lambda$, define $S f$ on $\mathscr{K}$ as

$$
(S f)\left(x_{1}, \ldots, x_{n}\right)=\sum_{p=0}^{k} \sum_{i_{1}=1}^{n} \ldots \sum_{i_{p}=1}^{n} f^{p}\left(x_{i_{1}}, \ldots, x_{i p}\right)
$$

the following sequence $\sum_{n=0}^{\infty} \mu_{\Lambda}^{n}(S f)$ converges (see also [3] where an analogous condition is imposed).

(iv) Finally a condition on the existence of the kinetic energy density; the following limit exists

$$
\lim _{\Lambda \rightarrow \infty} \sum_{n \geqq 0} \int \sum_{i=1}^{n} \frac{p_{i}^{2}}{2 m} \frac{d \mu_{\Lambda}^{n}\left(x_{1}, \ldots, x_{n}\right)}{V(\Lambda)}
$$

where $m$ is the mass of the particles and $V(\Lambda)=\int_{A} d x$. The limit $\Lambda \rightarrow \infty$ will always be understood in the sense of increasing cubes.

Our next task is to introduce a suitable class of homogeneous perturbed states. For each $\omega \in \tilde{\mathscr{F}}$ and $t$ element of $\mathbb{R}$ small enough (see later) we define a new state $\omega_{t}$ also belonging to $\widetilde{\mathscr{F}}$.

Let $u$ and $v$ be any pair of elements of $\mathscr{A}$ of the form

$$
u=\psi(S f) ; v=\phi(S g)
$$

where $\psi, \phi$ are real linear combinations of sin and $\cos$ on $\mathbb{R} ; f$ and $g$ elements of $\mathcal{O}$ with $q$-support in $\Lambda_{0}$.

For any $h \in \mathcal{O}$ with support in $\Lambda \times \mathbb{R}^{v}$ and for $t$ small enough, let

$$
\omega_{t}(W(h))=\sum_{n \geqq 0} \int d \mu_{\Gamma}^{n}(X) W(h)\left(X_{t}\right)
$$

where

$$
W(h)\left(X_{t}\right)=\exp i \sum_{k=1}^{n} h\left(q_{k}+t \int_{R^{\nu}} u_{a}(X) \frac{\partial}{\partial p_{k}} v_{a}(X) d a, p_{k}-t \int_{R^{\nu}} u_{a}(X) \frac{\partial}{\partial q_{k}} v_{a}(X) d a\right)
$$

and

$$
u_{a}(X)=u(X-a), v_{a}(X)=v(X-a),
$$

$\Gamma \supseteq \tilde{\Lambda}=\Lambda+\Gamma(0) ; \Gamma(0)$ is the unit cube in $R^{v}$ with center zero; the summations in the arguments of $h$ are understood to be component by component. 
Remark that

$$
\left|\int_{a}(X) \frac{\partial}{\partial p} v_{a}(X) d a\right| \leqq V\left(\Lambda_{0}\right)\|u\|\left\|\phi^{\prime}\right\|\left\|\frac{\partial g}{\partial p}\right\|=M
$$

where

$$
\begin{aligned}
& \|u\|=\sup _{x \in \mathscr{K}}|u(x)| \\
& \left\|\phi^{\prime}\right\|=\sup _{x \in \mathbb{R}}\left|\frac{d}{d x} \phi(x)\right| \\
& \left\|\frac{\partial g}{\partial p}\right\|=\sup _{p, q \in \mathbb{R}^{v}}\left|\frac{\partial p(q, p)}{\partial p}\right| .
\end{aligned}
$$

Analogously

$$
\left|\int u_{a}(X) \frac{\partial}{\partial q} v_{a}(X) d a\right| \leqq N
$$

where $M$ and $N$ are finite constants. Hence for $t$ satisfying

$$
0 \leqq|t|<\min (1 / N, 1 / M)
$$

each configuration $\left(x_{1}, \ldots, x_{n}\right) \notin\left(\tilde{\Lambda} \times \mathbb{R}^{v}\right)^{n} \quad$ yields $\quad\left(q_{1}+t \int u_{a} \frac{\partial}{\partial p_{1}} v_{a} d a, \ldots, p_{n}\right.$ $\left.-t \int u_{a} \frac{\partial}{\partial q_{n}} v_{a} d a\right) \notin\left(\Lambda \times \mathbb{R}^{v}\right)^{n}$

Therefore outside of the support of $W(h)$. By the compatibility condition of $\left(\mu_{\Lambda}^{n}\right)_{n}$, definition (2) is independent of $\Gamma$ and therefore defines a state on $\tilde{\mathscr{A}}$.

Lemma 2.1. For any $\omega \in \tilde{\mathscr{F}}$ and small $t \in R$, the state $\omega_{t}$ on $\mathscr{A}$ determined by (2) belongs to $\tilde{\mathscr{F}}$; the corresponding family of measures $\left(\mu_{t, \Lambda}^{n}\right)_{n \geqq 0}$ is defined by: for all $h \in \mathcal{O}$ with q-support in $\Lambda$,

$$
\sum_{n \geqq 0} \int d \mu_{t, \Lambda}^{n}(X) W(h)(X)=\sum_{n \geqq 0} \int d \mu_{\tilde{\Lambda}}^{n}(X) W(h)\left(X_{t}\right) .
$$

Proof. The family $\left(\mu_{t, \Lambda}^{n}\right)_{n \geqq 0}$ of density distributions is uniquely determined by the relation (5) as the induced cylinder measures which are also absolutely continuous with respect to the Lebesgue measure by (i). That the weight function is differentiable is ensured by the assumptions on $u$ and $v$.

As the perturbation is homogeneous, also $\omega_{t}$ is space translation invariant.

Using the boundedness of the functions in $\mathcal{O}$, condition (iii) is immediate.

Finally we verify (iv):

$$
\begin{aligned}
& \frac{1}{V(\Lambda)} \sum_{n \geqq 0} \int \sum_{i=1}^{n} p_{i}^{2} d \mu_{t, \Lambda}^{n}(X) \\
& =\frac{1}{V(\Lambda)} \sum_{n \geqq 0} \int \sum_{i=1}^{n}\left(p_{i}-t \int u_{a} \frac{\partial}{\partial q_{i}} v_{a} d a\right)^{2} d \mu_{\tilde{\Lambda}}^{n}(X)
\end{aligned}
$$




$$
\begin{aligned}
= & \frac{1}{V(\Lambda)} \sum_{n \geqq 0} \int d \mu_{\tilde{\Lambda}}^{n} p_{i}^{2}-\frac{2 t}{V(\Lambda)} \sum_{n \geqq 0} \int d \mu_{\tilde{\Lambda}}^{n} \sum_{i=1}^{n} p_{i} \int v_{a} \frac{\partial v_{a}}{\partial q_{i}} d a \\
& +\frac{t^{2}}{V(\Lambda)} \sum_{n \geqq 0} \int d \mu_{\tilde{\Lambda}}^{n} \sum_{i=1}^{n}\left(\int u_{a} \frac{\partial v_{a}}{\partial q_{i}} d a\right)^{2} .
\end{aligned}
$$

The first term converges as $\Lambda \rightarrow \infty$ by (iv).

Using the translation invariance and the compatibility of $\left(\mu_{A}^{n}\right)$, the second term converges to $-2 \mathrm{t} \omega\left(\Psi(S f) \phi^{\prime}(S g) S\left(p \frac{\partial g}{\partial q}\right)\right)$.

The third term equals

$$
\frac{t^{2}}{V(\Lambda)} \sum_{n \geqq 0} \int d \mu_{\tilde{\Lambda}}^{n} \sum_{i=1}^{n} \int d a\left[u \frac{\partial v}{\partial q_{i}} \int d b u_{b} \frac{\partial v_{b}}{\partial q_{i}}\right]_{a}
$$

and so converges - for the same reason as the second term-- to

$$
t^{2} \int \omega\left(\psi(S f) \psi(S f)_{a} \phi^{\prime}(S g) \phi^{\prime}(S g)_{a} S\left(\frac{\partial g}{\partial q} \frac{\partial g_{a}}{\partial q}\right)\right) d a
$$

where the last integral is over a bounded set.

For any pair $W(h)$ and $W(l)$ of generators of the algebra $\mathscr{A}$, their Poisson bracket is given by

$$
\{W(h), W(l)\}=i W(h+l) S\{h, l\}
$$

where $\{h, l\}(x)=\frac{\partial h}{\partial q} \cdot \frac{\partial l}{\partial p}-\frac{\partial h}{\partial p} \cdot \frac{\partial l}{\partial q}$.

Remark that the right hand side of formula (2) is differentiable with respect to $t$ at $t=0$ term by term.

After summation of the differentiated terms

$$
\left.\frac{d}{d t} \omega_{t}(W(h))\right|_{t=0}=\int \omega\left(u_{a}\left\{W(h), v_{a}\right\}\right) d a .
$$

Because of (iii) and

$$
\left|\int \omega\left(u_{a}\left\{W(h), v_{a}\right\}\right) d a\right| \leqq\|u\|\left\|\phi^{\prime}\right\| \int d a \omega\left(S\left|\left\{h, g_{a}\right\}\right|\right)
$$

formula (6) converges.

Finally we remark here that if the map $\omega \rightarrow \omega_{t}$ is formulated as a map of $\oplus^{\infty}\left(R^{2 v}\right)^{n}$ into itself, it is clear that it does not always represent a physical evolution because the local finiteness condition might be violated after an infinitely small time delay $t$.

\section{§3. Variational Principle and KMS-Condition}

We start with a formulation of the variational principle or the global thermodynamical equilibrium condition.

Suppose that for all $\Lambda \subset \mathbb{R}^{v}$, the local Hamiltonian $H_{A}$ with open boundary 
conditions is given by: for any configuration $X=\left(x_{1}, \ldots, x_{n}\right), x_{i} \in \Lambda \times \mathbb{R}^{v}$,

$$
H_{\Lambda}(X)=\sum_{x_{i}=\left(q_{i}, p_{i}\right) \in X} \frac{p_{i}^{2}}{2 m}+\sum_{Y \subseteq X} \phi(Y) .
$$

The interaction part is determined by the potential $\phi$, a real function on the non empty finite subsets of the physical space $\mathbb{R}^{v} ; \phi$ can be considered as a symmetric function on $\bigcup_{n \geqq 1}\left(\mathbb{R}^{v}\right)^{n}$ :

$$
\phi(X)=\phi\left(q_{1}, \ldots, q_{n}\right) .
$$

Furthermore we suppose

(i) translation invariance; for all $a \in \mathbb{R}^{v}: \phi(X)=\phi(X+a)$

(ii) at most $k$-particle interactions: $\phi\left(q_{1}, \ldots, q_{l}\right)=0$ if $l>k$

(iii) there exists finite sequence of functions $e(\phi)=\left(e^{1}, e^{2}, \ldots, e^{k}\right)$; the $e^{i}$ are real bounded differentiable functions on $\left(\mathbb{R}^{2 v}\right)^{i}$ with compact support and such that for $\Lambda$ large enough

$$
\sum_{Y \subseteq X} \phi(Y)=\int_{\Lambda} d a \operatorname{Se}(\phi)(X-a) .
$$

The motivation for these conditions is found in [9] and [8] where they are proved to hold for bounded pair potentials respectively for the hard core system. However we have the more restrictive condition of finite range interaction.

Remark further that the kinetic energy $T_{A}$ :

$$
T_{\Lambda}(X)=\sum_{x_{i}=\left(q_{i}, p_{i}\right) \in X} p_{i}^{2} / 2 m
$$

also can be written as follows: for $\Lambda$ large enough up to boundary effects,

$$
T_{\Lambda}(X)=\int_{\Lambda} d a(S t)(X+a)
$$

where $t(q, p)=\varepsilon(q) p^{2} / 2 m$, and $\varepsilon$ is a $C^{\infty}$-function on $\mathbb{R}^{v}$ with support in $\Gamma(0)$ such

that $\int_{\Gamma(0)} \varepsilon(q) d q=1$.

Conditions (iii) and (iv) imply now:

Condition 3.1. We restrict $\widetilde{\mathscr{F}}$ to the states $\omega$, for which the following limit exists

$$
\lim _{\Lambda \rightarrow \infty} \frac{\omega\left(H_{\Lambda}\right)}{V(\Lambda)}=\omega(S(t+e(\phi))) .
$$

This set is also closed under the perturbations.

Definition 3.2. Let $\omega$ be an element of $\widetilde{\mathscr{F}}$ and $\phi$ an interaction as defined above; and $s(\omega)$ finite, where $s(\omega)$ is the entropy density of the state $\omega$ [14] given by

$$
s(\omega)=\lim _{\Lambda \rightarrow \infty} \inf \frac{S_{\omega}(\Lambda)}{V(\Lambda)}
$$

$\omega$ is called globally thermodynamically stable (G.T.S.) at inverse temperature $\beta$ if for all $\rho \in \widetilde{\mathscr{F}}$ :

$$
\beta \rho(S(t+e(\phi)))-s(\rho) \geqq \beta \omega(S(t+e(\phi)))-s(\omega)
$$




$$
S_{\omega}(\Lambda)=-\sum_{n \geqq 0} \frac{1}{n !} \int d X \sigma_{\Lambda}^{n}(X) \log \sigma_{\Lambda}^{n}(X) .
$$

Remark that, if $X \subset\left(\Lambda \times \mathbb{R}^{v}\right)^{n}$ then for all $\Lambda^{\prime} \supset \Lambda$ the expression $\left\{H_{\Lambda^{\prime}}, W(h)\right\}(X)$, $h \in \mathcal{O}$ is independent of $\Lambda^{\prime}$, expressed by

$$
\{H, W(h)\}(X)=\lim _{\Lambda \rightarrow \infty}\left\{H_{\Lambda}, W(h)\right\}(X) .
$$

Furthermore $\omega(W(f)\{H, W(g)\}), f, g \in \mathcal{O}$ exists as can be checked analogously as was done for formula (6).

Definition 3.3. Any state $\omega \in \tilde{\mathscr{F}}$ is said to satisfy the static $\mathrm{KMS}$-condition $[1,2,3,4]$ at inverse temperature $\beta$ for a Hamiltonian $H$ if for all $f, g \in \mathcal{O}$ :

$$
\beta \omega(W(f)\{H, W(g)\})=\omega(\{W(f), W(g)\}) .
$$

Now we formulate our main result:

Theorem 3.4. If $\omega \in \tilde{\mathscr{F}}$ satisfies GTS (Definition 3.2) then $\omega$ satisfies KMS (Definition 3.3) for the same temperature and the same interaction.

The proof of this theorem is based on the following steps.

Lemma 3.5. With $\omega_{s}, s \in \mathbb{R}$ defined as in (2):

$$
\lim _{s \rightarrow 0} \frac{\left(\omega_{s}-\omega\right)}{s}(S(t+e(\phi)))=\omega(u\{H, v\}) .
$$

Proof. From (6) and condition (iii) and (iv) on $\omega$

$$
\begin{aligned}
& \lim _{s \rightarrow 0} \frac{\left(\omega_{s}-\omega\right)}{s}(S(t+e(\phi))) \\
& =\int \omega\left(u_{a}\left\{S(t+e(\phi)), v_{a}\right\}\right) d a .
\end{aligned}
$$

Using the spatial invariance of $\omega$ and the relations (8) and (9) we get the lemma.

Lemma 3.6. With the notations of above:

$$
\delta s(\omega)=\liminf _{t \rightarrow 0^{+}} \frac{s\left(\omega_{t}\right)-s(\omega)}{t} \geqq \omega(\{u, v\}) .
$$

Proof. Consider $(\Gamma(m))_{m \geqq 0}$ an increasing absorbing set of cubes of $\mathbb{R}^{v}$. First suppose that $\omega$ is such that for all $n$ and $\Lambda, \sigma_{\Lambda}^{n}(x)>0$ for all $x \in\left(\Lambda \times \mathbb{R}^{v}\right)^{n}$. Using the definition of the entropy density (Definition 3.2)

$$
\begin{aligned}
& \delta s(\omega)=\liminf _{t \rightarrow 0^{+}} \frac{s\left(\omega_{t}\right)-s(\omega)}{t} \\
& =\liminf _{t \rightarrow 0^{+}} \frac{1}{t}\left[\liminf _{m \rightarrow \infty} \frac{S \omega_{t}(\Gamma(m))}{V(\Gamma(m))}+\limsup _{m}-\frac{S_{\omega}(\Gamma(m))}{V(\Gamma(m))}\right] .
\end{aligned}
$$

There exists a subsequence $\left(\Gamma\left(m_{\alpha}\right)\right)_{m_{\alpha}}$ such that

$$
\limsup _{m_{\alpha} \rightarrow \infty} \frac{S \omega_{t}\left(\Gamma\left(m_{\alpha}\right)\right)}{\Gamma\left(m_{\alpha}\right)}=s\left(\omega_{t}\right) .
$$


hence

$$
\begin{gathered}
\delta S(\omega) \geqq \liminf _{t \rightarrow 0^{+}} \limsup _{m_{\alpha} \rightarrow \infty} \frac{S \omega_{t}\left(\Gamma\left(m_{\alpha}\right)\right)-S_{\omega}\left(\Gamma\left(m_{\alpha}\right)\right)}{t V\left(\Gamma\left(m_{\alpha}\right)\right)} \\
=\liminf _{t \rightarrow 0^{+}} \limsup _{m_{\alpha} \rightarrow \infty}\left\{-\lim _{N \rightarrow \infty} \sum_{n=0}^{N} \int_{\left(\Gamma\left(m_{\alpha}\right) \times \mathbb{R}\right)^{n}} d x\left[\sigma_{t, \Gamma\left(m_{\alpha}\right)}^{n}(x)\right] \log \sigma_{t, \Gamma\left(m_{\alpha}\right)}^{n}\right. \\
\left.\left.\quad-\sigma_{\Gamma\left(m_{\alpha}\right)}^{n}(x) \log \sigma_{\Gamma\left(m_{\alpha}\right)}^{n}(x)\right] \frac{1}{t V\left(\Gamma\left(m_{\alpha}\right)\right)}\right\} .
\end{gathered}
$$

There exists a net $\left(\Omega_{n, j}\right)_{j}$ of compact subsets of $\left(\Gamma\left(m_{\alpha}\right) \times \mathbb{R}^{v}\right)^{n}$ such that

$$
\int_{\left(\Gamma\left(m_{\alpha}\right) \times \mathbb{R}^{v}\right)^{n}} d x[\ldots]=\lim _{\Omega_{n, j} \rightarrow\left(\Gamma\left(m_{\alpha}\right) \times \mathbb{R}^{v}\right)^{n}} \int_{\Omega_{n, j}} d x[\ldots] .
$$

As we supposed that the weight functions are strictly positive on compact sets the function

$$
s \rightarrow \int_{\Omega_{n, j}} d x \sigma_{s, \Gamma\left(m_{\alpha}\right)}^{n}(x) \log \sigma_{s, \Gamma\left(m_{\alpha}\right)}^{n}(x)
$$

is differentiable, and using $\frac{d \sigma_{t, \Gamma\left(m_{\alpha}\right)}^{n}(x)}{d t}=\int d a\left\{v_{a}, u_{a} \sigma_{t, \Gamma\left(m_{\alpha}\right)}^{n}\right\}(x)+t C^{n}(Y)$ for some $Y$ between $x$ and $x_{t}$, obtained from (6), after partial integration, $C^{n}$ is obtained from the mean value theorem. Because of (3), (4): $\left|C^{n}(Y)\right| \leqq n P$ where $P$ is a constant. As the correction term vanishes if $\Gamma\left(m_{\alpha}\right) \rightarrow \infty$ in (10), from now on we disregard this contribution, and:

$$
\begin{aligned}
& \lim _{\Omega_{n, j} \rightarrow\left(\Gamma\left(m_{\alpha}\right) \times \mathbb{R}^{v}\right)^{n}}\left\{\int_{\Omega_{n, j}} d x[\ldots]\right\} \\
& =\lim _{\Omega_{n, j}} \frac{1}{t} \int_{0}^{t} d s \frac{d}{d s} \int_{\Omega_{n, j}} d x \sigma_{s, \Gamma\left(m_{\alpha}\right)}^{n}(x) \log \sigma_{s, \Gamma\left(m_{\alpha}\right)}^{n}(x) \\
& =\lim _{\Omega_{n, j}} \frac{1}{t} \int_{0}^{t} d s \int d x \int d a\left[\left\{v_{a}, u_{a} \sigma_{s, \Gamma\left(m_{\alpha}\right)}^{n}\right\}(x) \log \sigma_{s, \Gamma\left(m_{\alpha}\right)}^{n}(x)\right. \\
& \left.\quad+\left\{v_{a}, u_{a} \sigma_{s, \Gamma\left(m_{\alpha}\right)}^{n}\right\}\right] .
\end{aligned}
$$

The second term of this expression adds up to $\frac{d}{d s} \omega_{s}(1)=0$. Therefore we drop it from now on.

Due to the compact support of $f$ and $g$ (see (1)) for $\Omega_{n, j}$ large enough the right hand side of (11) is independent of this domain of integration. Remark that all integrals are over compact sets, such that the orders of integration can be interchanged. Then performing partial integration we get, using (10) and (11):

$$
\delta S(\omega) \geqq \liminf _{t \rightarrow 0^{+}} \limsup _{m_{\alpha} \rightarrow \infty} \lim _{N \rightarrow \infty} \sum_{n=0}^{N}-\frac{1}{n !} \frac{1}{t} \int_{0}^{t} d s \int d a \int d x \frac{\sigma_{s, \Gamma\left(m_{\alpha}\right)}^{n}\left\{u_{a}, v_{a}\right\}(x)}{V\left(\Gamma\left(m_{\alpha}\right)\right)} .
$$


Using the compatibility and the translation invariance of the states:

$$
\begin{aligned}
& \delta s(\omega) \geqq \liminf _{t \rightarrow 0^{+}} \limsup _{m_{\alpha} \rightarrow \infty} \lim _{N} \sum_{n=0}^{N} \frac{1}{n !} \int_{t}^{t} d s \\
& {\left[\frac{V\left(S\left(m_{\alpha}\right)\right)}{V\left(\Gamma\left(m_{\alpha}\right)\right)} \int d x \sigma_{s, A_{0}}^{n}(x)\{u, v\}(x)\right.} \\
& \left.\quad+\frac{1}{V\left(\Gamma\left(m_{\alpha}\right)\right)} \int_{R\left(m_{\alpha}\right)} d a \int d x \sigma_{s, \Gamma\left(m_{\alpha}\right)}^{n}(x)\left\{u_{a}, v_{a}\right\}(x)\right]
\end{aligned}
$$

where $S\left(m_{\alpha}\right)=\left\{a \in \Gamma\left(m_{\alpha}\right)-\Lambda_{0} \mid \Gamma\left(m_{\alpha}\right)-a \supseteq \Lambda_{0}\right\}$ and $R\left(m_{\alpha}\right)$ is the complement of $S\left(m_{\alpha}\right)$ in $\Gamma\left(m_{\alpha}\right)-\Lambda_{0}$. Remark that

$$
\begin{aligned}
& \lim _{m_{\alpha}} \frac{V\left(S\left(m_{\alpha}\right)\right)}{V\left(\Gamma\left(m_{\alpha}\right)\right)}=1 . \\
& \lim _{m_{\alpha}} \frac{V\left(R\left(m_{\alpha}\right)\right)}{V\left(\Gamma\left(m_{\alpha}\right)\right)}=0
\end{aligned}
$$

therefore by condition (iii) only the first term survives and

$$
\delta s(\omega) \geqq \liminf _{t \rightarrow 0^{+}} \sum_{n=0}^{\infty} \frac{1}{n !} \frac{1}{t} \int_{0}^{t} d s \int d x \sigma_{s, A_{0}}^{n}(x)\{u, v\}(x) .
$$

The lemma is proved if we prove that the map $s \rightarrow \omega_{s}(\{u, v\})$ is continuous. This follows from:

$$
\begin{aligned}
& \left|\left(\omega_{t}-\omega_{s}\right)(\{u, v\})\right| \leqq \sum_{n=N}^{\infty}\left|\int d \mu_{\Lambda}^{n}(x)\{u, v\}\left(x_{t}\right)\right|+\left|\sum_{n=N}^{\infty} \int d \mu_{\Lambda}^{n}(x)\{u, v\}\left(x_{s}\right)\right| \\
& +\sum_{n=0}^{N-1} \int d \mu_{\Lambda}^{n}(x)\left|\{u, v\}\left(x_{t}\right)-\{u, v\}\left(x_{s}\right)\right| .
\end{aligned}
$$

The first and second term are small for $N$ large enough independent of $t$ and $s$ in some small interval around zero by (iii) and the boundedness of the derivatives of $\psi, \phi, f$ and $g$. The last term is small for $|t-s|$ small enough by the uniform continuity of $\{u, v\}(x), x=\left(x_{1}, \ldots, x_{n}\right), n<N$.

Finally we eliminate the positivity condition on the weight functions. If $\omega$ does not have this positivity property consider then the state $\omega^{\tau}=(1-\tau) \omega+\tau \rho$; $\tau \in(0,1)$

where $\rho$ is the state with weight functions (see [13])

$$
\sigma_{\Lambda}^{n}(x)=\exp \left[-V(\Lambda) \pi^{n v / 2}\right] \exp \left[-\sum_{i=1}^{n} p_{i}^{2}\right] .
$$

It is clear that $\rho \in \tilde{\mathscr{F}}$ and that the state $\omega^{\tau}$ has the positivity property for all $\tau \in(0,1)$. From the above argument we have

$$
\delta s\left(\omega^{\tau}\right) \geqq \omega^{\tau}(\{u, v\}) ; \tau \in(0,1) .
$$


As the entropy density is affine [14] we get

$$
(1-\tau) \delta s(\omega)+\tau \delta s(\rho) \geqq(1-\tau) \omega(\{u, v\})+\tau \rho(\{u, v\})
$$

Because $\delta s(\rho)$ is finite, we get the result, letting $\tau$ tend to zero.

Proof of Theorem 3.4. Suppose that $\omega$ satisfies GTS, then for $\omega_{s}$ as in (2)

$$
\liminf _{s \rightarrow 0^{+}} \frac{\beta\left(\omega_{s}-\omega\right)}{s}(S(t+e(\phi)) \geqq \delta s(\omega)
$$

by Lemma 3.5. and 3.6. we get

$$
\beta \omega(u\{H, v\}) \geqq \omega(\{u, v\}) .
$$

As this also holds for $u$ replaced by $-u$, we get the equality

$$
\beta \omega(u\{H, v\})=\omega(\{u, v\}) .
$$

Choose subsequently $u=\cos S f, \sin S f$ and $v=\cos S g, \sin S g$. By linearity of the Equation (12) we get the theorem.

Acknowledgement. The authors thank Professor H. Araki for numerous suggestions to improve the paper.

\section{References}

1. Kubo, R. : J. Phys. Soc. Japan 12, 570 (1957)

2. Gallavotti, G., Verboven, E. : Nuovo Cimento 28, 274 (1975)

3. Aizenman, M., Goldstein, S., Gruber, C., Lebowitz, J. L., Martin, P. : Commun. math. Phys. 53, 209 (1977)

4. Aizenman, M., Gallavotti, G., Goldstein, S., Lebowitz, J. L. : Commun. math. Phys. 48, 1 (1976)

5. Haag, R., Kastler, D., Trych-Pohlmeyer, E. : Commun. math. Phys. 38, 173 (1974)

6. Lanford, D. E., Ruelle, D.: Commun. math. Phys. 13, 194 (1969)

7. Ruelle, D. : Commun. math. Phys. 18, 127 (1970)

8. Gallavotti, G., Miracle-Sole, S. : Ann. Inst. Henri Poincaré 8, 287 (1968)

9. Gerardi, A., Ianiro, N., Maffei, C., Marchioro, C. : J. Math. Phys. 16, 438 (1975)

10. Verbeure, A. : Algebras of operators and their applications to mathematical physics. Proceedings on the Marseille Conference, 1977 (ed. D. Kastler, D.W. Robinson). (to appear)

11. Fannes, M., Verbeure, A. : J. Math. Phys. 19, 558 (1978)

12. Ruelle, D. : Statistical mechanics. New York: Benjamin 1969

13. Pulè, J. V., Verbeure, A. : J. Math. Phys. 18, 862 (1977)

14. Robinson, D., Ruelle, D. : J. Math. Phys. 5, 288 (1967)

Communicated by $\mathrm{H}$. Araki

Received November 11, 1977; in revised form March 27, 1978 